\title{
Solar Variability Traced by Cosmogenic Isotopes
}

\section{By J ÜR BEER ${ }^{1}$, STEPHAN T. BAUMGART NER ${ }^{1}$, BEATE DIT TRICH-H A N NEN ${ }^{2}$, JÜRG HAUENSTEIN ${ }^{3}$, PETER KUBIK CHRISTIAN LUKASCZYK ${ }^{1}$, WERNER MENDE
RITA STELLMACHER AND MARTIN SUTER}

\author{
${ }^{1}$ Swiss Federal Institute for Environmental Science and Technology (EAWAG), \\ CH-8600 Dübendorf, Switzerland \\ ${ }^{2}$ Institute of Particle Physics, ETH-Hönggerberg, CH-8093 Zürich, Switzerland \\ ${ }^{3}$ Physics Institute, University of Bern, CH-3012 Bern, Switzerland \\ ${ }^{4}$ Paul Scherrer Institute, c/o Institute of Particle Physics, ETH-Hönggerberg, \\ CH-8093 Zürich, Switzerland \\ ${ }^{5}$ Institute of Meteorology, Freie Universität Berlin, Germany
}

\begin{abstract}
${ }^{10} \mathrm{Be}$ has been measured in a $300 \mathrm{~m}$ long ice core from Greenland. After a general analysis of the
${ }^{10} \mathrm{Be}$ data the potential and the limitations of ${ }^{10} \mathrm{Be}$ records in ice to reconstruct solar variability are discussed. Special emphasis is given to periods of low solar activity (Maunder Minimum etc.). The 'clock problem' is addressed, also the question whether the Schwabe cycle maintains its phase. Finally the question of what ${ }^{10} \mathrm{Be}$ can tell us about changes of the solar irradiance in the past is explored.
\end{abstract}

\section{Introduction}

Although the existence of sunspots has been known for a long time, it was not before 1843 that the cyclicity of sunspot occurences was recognised by Heinrich Schwabe. His discovery of the 11-year or better Schwabe cycle 150 years ago was the beginning of continuous systematic observations of solar variability. Other parameters like changes in the solar diameter or terrestrial occurences like auroras and magnetic disturbances at antipodal stations (aa index) are all related to the magneto-hydrodynamic processes taking place in the convective zone of the Sun. In recent years the Sun has been continuously monitored by Earth and satellite based instruments. Probably the most important result of the last 15 years is the observation of a positive correlation between the solar irradiance and the sunspot number (solar activity). The irradiance exhibits variations of about $0.1 \%$ during one cycle (Willson \& Hudson 1988). According to the standard solar model the energy production by fusion is extremely stable over periods of centuries or millennia. Therefore, variations of solar irradiance should only be attributed to fluctuations of the energy transport within the convective zone and to sunspot blocking effects in the photosphere which have to be compensated after some time. Since the Sun represents the engine driving the climate system of the Earth, any variation of the solar irradiance is directly reflected in a change of solar forcing which in turn influences climate. Although solar variability over the last decade was rather small, a fundamental question arises: How large was the solar variability in the past and how large will it be in the future? Based on the level of variability observed for Sun-like stars the potential of the Sun seems to be rather large (Baliunas \& Jastrow 1990).

When studying solar variability the main problem is that in spite of an almost complete set of direct observations of all emitted radiation and particles this information is 
restricted to a very short time period of decades. This time scale is by far not sufficient to investigate the long-term solar variability. The longest record of continuous, direct observations is the sunspot record. It goes back, with decreasing reliability, to about 1700 A.D.. Before the Maunder Minimum period (1645 - 1715 A.D.) there is a lack of consistent records of directly observed solar variability. Several attempts have therefore been made to develop indirect methods with which the history of solar variability can be reconstructed. The search for solar variability in various natural archives such as tree rings, sediments and ice cores turned out to be a rather difficult task due to the smallness of the effects induced by solar variability.

Probably the most successful indirect tool so far for establishing the history of solar variability is the use of cosmogenic radioisotopes (Raisbeck \& Yiou 1980). Since their production rate is modulated by the interaction of the solar wind with the primary cosmic ray particles, they provide information which can be attributed to solar variability. Records of counters monitoring secondary cosmic ray particles since 1937 show a clear anticorrelation between the cosmic ray flux impinging on the Earth's atmosphere and the solar activity expressed in sunspot numbers (Beer et al. 1991). The main two cosmogenic isotopes used so far are ${ }^{14} \mathrm{C}$ and ${ }^{10} \mathrm{Be}$. Due to their different geochemical behaviour they are somewhat complementary. ${ }^{14} \mathrm{C}$ oxidises to ${ }^{14} \mathrm{CO}_{2}$ after production and then exchanges between the main carbon reservoirs atmosphere, ocean and biosphere. Due to the large size of these reservoirs short-term variations are strongly dampened and delayed. On the other hand the atmospheric residence time is longer than the corresponding mixing time leading to a global mean value.

The main archive for ${ }^{14} \mathrm{C}$ is tree rings which have been analysed back to $\sim 11,000$ years B.P. with a resolution of 20 years and for some periods even with one year (Stuiver et al. 1991). The absolute time scale is obtained by counting the rings.

${ }^{10} \mathrm{Be}$ attaches itself to aerosols and is removed from the atmosphere mainly by wet deposition within about 1.5 years after its production. As a consequence, the deposition flux at a specific site does not necessarily represent the global mean value. It contains also a local component which can be related to atmospheric processes (e.g. exchange stratosphere - troposphere, precipitation rate).

On the other hand, due to its short atmospheric residence time ${ }^{10} \mathrm{Be}$ is also sensitive to short-term production variations, such as expressed in the Schwabe cycle. Based on model calculations production variations of $30-50 \%$ are to be expected during a cycle depending on the mean level of solar activity. In the case of ${ }^{14} \mathrm{C}$ these variations are attenuated by about a factor of 100 (Siegenthaler \& Beer 1988).

The best archive for ${ }^{10} \mathrm{Be}$ measurements are ice sheets which directly store the atmospheric fall-out. However, $1 \mathrm{~kg}$ of ice contains only about $10^{7}$ atoms ${ }^{10} \mathrm{Be}$ which makes detection by decay counting impossible. With the development of accelerator mass spectrometry such small numbers of atoms became measurable.

Newkirk (1984) discussed the potential of this technique in a paper entitled: "What can accelerator mass spectrometry do for solar physics?". Some of the questions he raised were: 1 . Does the 11-year cycle continue through the Maunder, Spoerer, and other past minima in the envelope of solar activity? 2. Does the cycle maintain phase through such minima? 3. Does solar activity follow an 80-90 year variation as suggested by the more limited optical and auroral records? 4. Is the modern character of the 11-year cycle typical of eras of high solar activity in the past? 5 . Are climatological variations in any way induced or affected by solar variations? 6. What is the power spectrum of solar activity?

In search for answers to the above questions, we now discuss a continuous annual ${ }^{10} \mathrm{Be}$ record covering the last 563 years. 


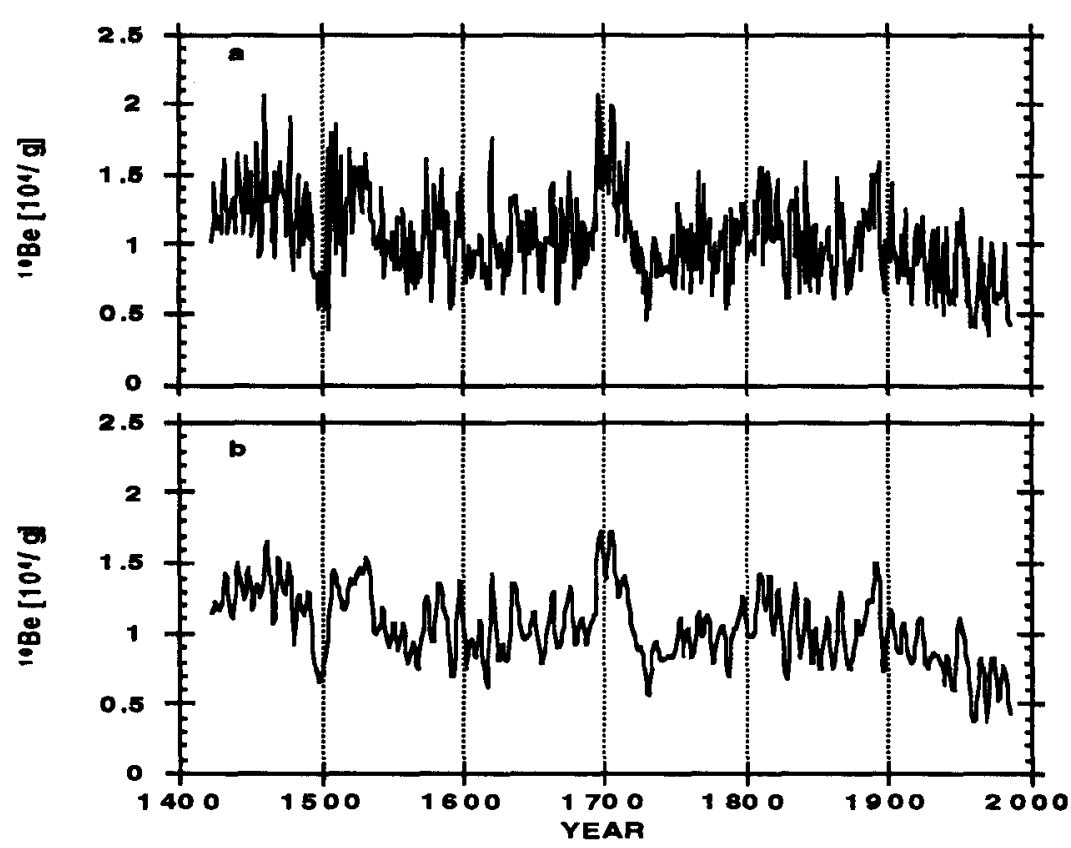

Figure 1. (a) Annual ${ }^{10}$ Be concentration in $10^{4}$ atoms per gram ice after interpolation of the measured data using cubic splines. The relative precision $(1 \sigma)$ of the measurements is $5-8 \%$. (b) ${ }^{10} \mathrm{Be}$ concentration of the same data after applying a low-pass filter with a cut-off of 6 years.

\section{Experimental}

The analysed ice core was drilled at Dye $3\left(65.2^{\circ} \mathrm{N} ; 43.8^{\circ} \mathrm{W}\right)$, Greenland. It has a length of $300 \mathrm{~m}$ corresponding to 563 years. The ${ }^{10} \mathrm{Be}$ results of the upper part dating as far back as 1783 A.D. have been published earlier (Beer et al. 1990). Dating of the upper part is based on determinations of annual layer thicknesses by measuring $\mathrm{H}_{2} \mathrm{O}_{2}$ which shows strong seasonality. Before 1783 the core was cut into $50 \mathrm{~cm}$ and further down into $40 \mathrm{~cm}$ long pieces which is close to the annual accumulation. The time scale was established afterwards using $\delta^{18} \mathrm{O}$ variations on the nearby deep-drilling core (Clausen, personal communication) assuming that the annual layers do not differ significantly between the two cores. Each sample of about $2 \mathrm{~kg}$ of ice was prepared following standard procedures and was analysed at the AMS facility of ETH/PSI Zürich (Suter 1990 ). ${ }^{36} \mathrm{Cl}$ measurements corresponding to the ${ }^{10} \mathrm{Be}$ data presented here are currently being carried out.

\section{Results and discussion}

\subsection{General analysis of the data}

The measured data which cover the period from $1423 \mathrm{AD}$. to $1985 \mathrm{AD}$. have been interpolated using natural cubic splines to obtain equidistant annual data (Figure 1(a)). To remove the rather large short-term fluctuations we applied a low-pass filter with a cut-off frequency of $0.164 \mathrm{y}^{-1}$. (Figure $1(b)$ ). The data between 1783 and 1985 have been discussed elsewhere (Beer et al. 1990). Figure 1(a) shows that annual variations of ${ }^{10} \mathrm{Be}$ concentrations are rather large. We attribute this to fluctuations of transport 


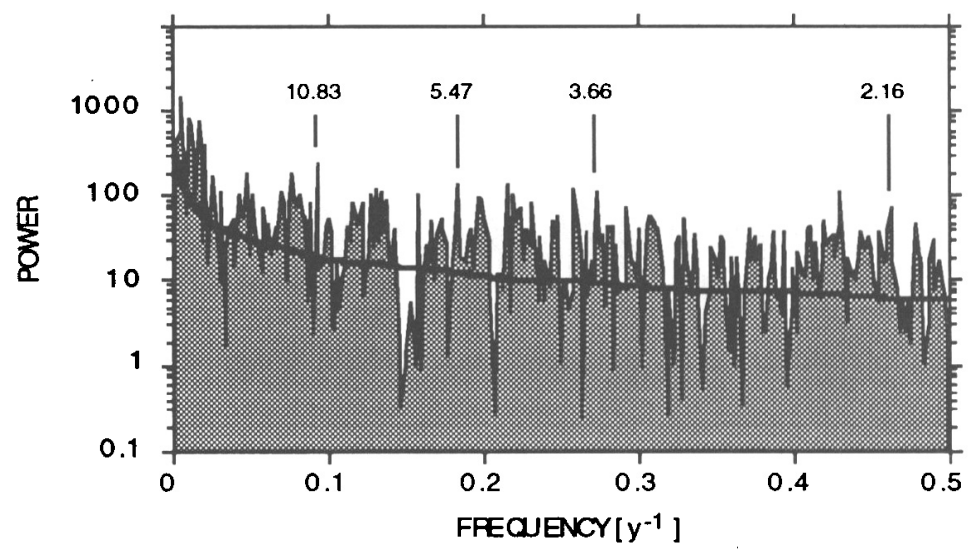

FIgURE 2. DFT-Fourier spectrum of the data of Figure 1(a). The solid line represents a hyperbolic model spectrum. The most prominent peak below $45 \mathrm{y}$ corresponds to the $10.83 \mathrm{y}$ Schwabe cycle. Its second, third and fifth harmonic can also be seen.

and deposition processes, because annual variations of the production rate are smaller $(<50 \%$ ) (Blinov 1988). These short-term variations are superimposed on a long termtrend with maxima near 1500,1700 and 1800 . The 20 th century is characterised by a decrease of the ${ }^{10} \mathrm{Be}$ concentration. The filtration in Figure $1(b)$ reveals strong, nearly decadal oscillations which we relate to the solar Schwabe cycle.

Figure 2 displays the results of a DFT-Fourier analysis of the 563 data points shown in Figure 1(a) using a rectangular window. One of the most prominent peaks in the spectrum corresponds to a period of 10.83 years. It raises $9 \mathrm{~dB}$ above the fitted hyperbolic model spectrum. The second, third and fifth harmonics of this peak are also clearly visible $(5.47,3.66,2.16$ years). The deviations from the theoretical harmonic frequencies are small $(-0.98 \%,-1.33 \%$ and $0.29 \%)$ and the phases are coherent indicating a common source of the signal. Precise determination of long periods is difficult due to the limited length of the record. Therefore, the transformation window has been extended by means of spline embedding. The Fourier spectrum of this extended series shows among others $(60.86,46.92$ and 20.97 years) a spectral peak at 87.4 years which can be attributed to the Gleissberg-cycle.

The coherence of the record was tested by calculating an average spectrum. A window of 256 years was moved in steps of 30 years across the data interval yielding 12 Fourier spectra. Averaging of these spectra reveals again a peak at 10.85 years (including the second, third and fifth harmonic) in good agreement with the single spectrum of the whole ${ }^{10} \mathrm{Be}$ record.

\section{3:2. ${ }^{10}$ Be and solar variability}

There are strong, but complex couplings between the ${ }^{10} \mathrm{Be}$ production rate and the magnetic properties of the solar wind which itself is related to various solar processes and their manifestations as observed from the Earth. The most famous phenomenon is the sunspot cycle. However, it seems that there are periods when this cycle almost disappears (Maunder Minimum). Other more recently observed phenomena related to the Schwabe cycle are fluctuations in the number of flares, plages, variations of the solar diameter and changes in the emission of solar wind. When the solar wind interacts with the Earth it causes fluctuations of the geomagnetic field strength (aa index), induces ionisation 


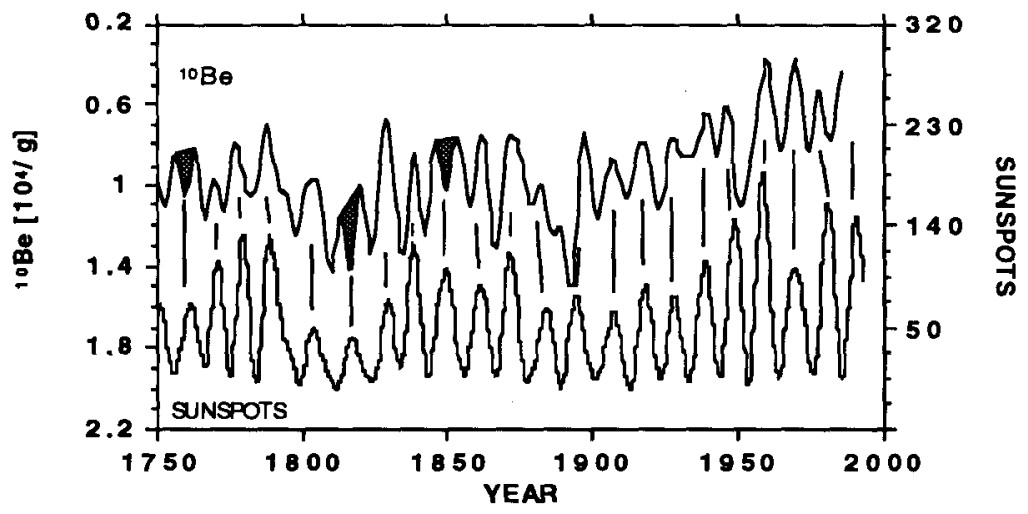

Figure 3. Comparison of the filtered ${ }^{10} \mathrm{Be}$ data of Figure $1(b)$ with the sunspot record. The correspondence between sunspot maxima and ${ }^{10} \mathrm{Be}$ minima is indicated by lines. Three sunspot maxima coincide with local ${ }^{10} \mathrm{Be}$ maxima (shaded).

processes in the upper atmosphere leading to auroras, and modulates the production rate of cosmogenic radioisotopes like ${ }^{10} \mathrm{Be}$ and ${ }^{14} \mathrm{C}$. Finally, satellite measurements show changes in spectral and the total solar irradiance depending on the sunspot numbers and the intensity of the magnetic network (Lean 1991). Although all these effects are in the end related to the magneto-hydrodynamical processes taking place in the Sun's convective zone, their relationship is not yet well-known. It seems that they are not linearly coupled as far as amplitudes and phases are concerned. The situation is complicated by the fact that some of the parameters show only very small fluctuations and are strongly linked (solar diameter, total irradiance) or are influenced by additional parameters (cosmogenic isotopes). The longest direct record of solar variability, so far, is the sunspot record. Systematic observations began in 1826 . The period from approximately $1720-1826$ has been reconstructed from various individual observations. Going further back in time data become sparse and more and more unreliable. Nevertheless, the 17th century was obviously a very quiet period regarding sunspots (Eddy 1976). Since all other records of solar activity are much shorter (aa index, solar diameter and irradiance) or less reliable (aurora) we concentrate here on a comparison between ${ }^{10} \mathrm{Be}$ and the sunspot data.

A discussion of the modulation of the production rate of ${ }^{10} \mathrm{Be}$ is given by Lal \& Peters (1967).

In Figure 3 the filtered ${ }^{10} \mathrm{Be}$ record from Figure $1(b)$ is compared with the sunspot record for the period $1750-1985$. Since large sunspot numbers correspond to high solar activity leading to a reduction of the ${ }^{10} \mathrm{Be}$ production rate, the ${ }^{10} \mathrm{Be}$ scale is inverted. As indicated by the lines in Figure 3,12 peaks are synchronous within one year but some are shifted up to 4 years. All the sunspot maxima can be related to corresponding ${ }^{10} \mathrm{Be}$ minima except at three points $(1760,1820,1850)$ where ${ }^{10} \mathrm{Be}$ shows an additional maximum not represented in the sunspot record. These peaks can be interpreted either as real in the sense that they are the result of solar modulation not visible in the sunspot numbers or as a consequence of terrestrial transport and deposition processes. In the first case this would indicate that there are solar processes which lead to changes in the solar wind but do not influence the sunspot number. This seems rather unlikely, although during the Maunder Minimum we have a situation where we observe ${ }^{10} \mathrm{Be}$ variations in spite of the missing sunspots. In the second case we need additional information to separate production and transport effects. One possibility is to combine the ${ }^{10} \mathrm{Be}$ record 


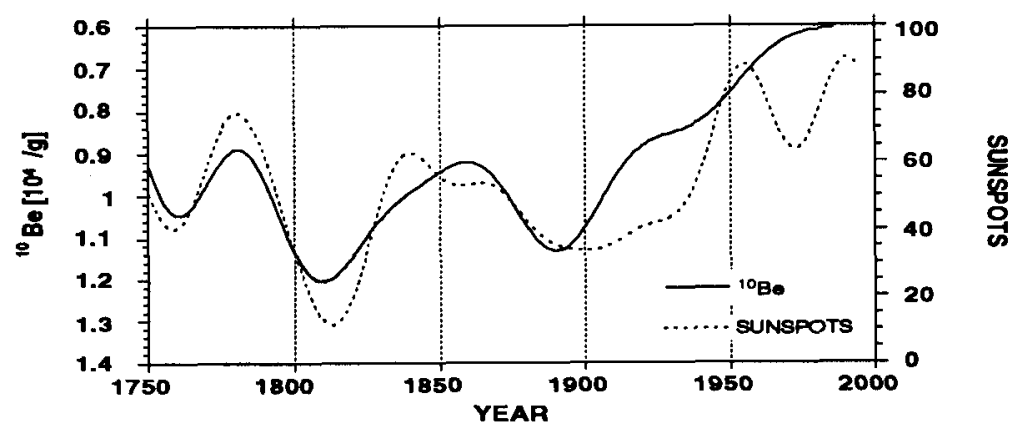

FIgURE 4. Long-term trends of the ${ }^{10} \mathrm{Be}$ concentration and the sunspot number after applying spectral low-pass filters ( $41 \mathrm{y}$ for ${ }^{10} \mathrm{Be}, 31 \mathrm{y}$ for sunspots).

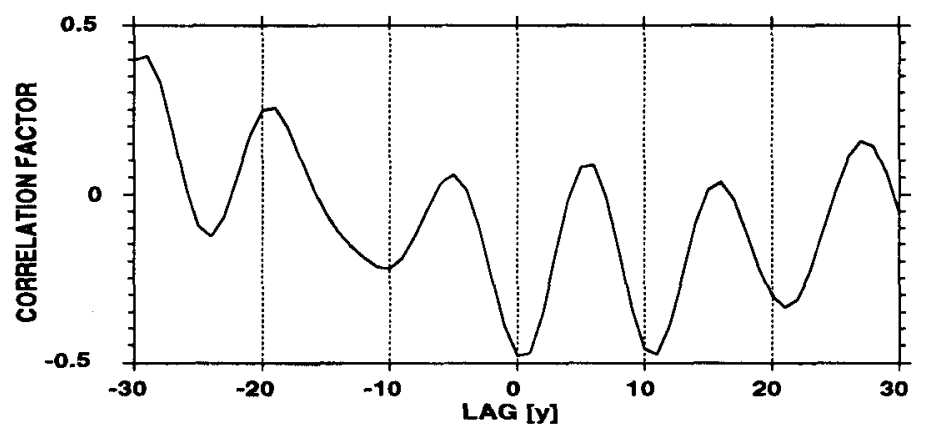

FIGURE 5. Cross correlation function between the ${ }^{10} \mathrm{Be}$ concentration and the sunspot number shown in Figure 3 . The highest correlation of -0.46 corresponds to a ${ }^{10} \mathrm{Be}$ lag of $0.5 \mathrm{y}$.

with the ${ }^{36} \mathrm{Cl}$ record of the same samples. Due to the different geochemical behaviour of ${ }^{10} \mathrm{Be}$ and ${ }^{36} \mathrm{Cl}$, transport and deposition effects should at least be partly removable. The second possibility is to combine the ${ }^{10} \mathrm{Be}$ record with a second ${ }^{10} \mathrm{Be}$ record from another site. This procedure has been successfully applied earlier (Beer et al. 1991).

Figure 4 shows the long-term trends obtained by low-pass filters (41 years for ${ }^{10} \mathrm{Be}, 31$ years for sunspots). Both curves show the same features, namely the Dalton solar minimum (1795-1830 A.D.) and an increasing long-term trend during the 20th century. The cross-correlation of the data of Figure 3 is displayed in Figure 5. The largest correlation of -0.46 is obtained for $\mathrm{a}^{10} \mathrm{Be}$ lag of 0.5 years which is shorter than the 1.5 years found for the period 1783 - 1985 (Beer et al. 1990). The oscillations of the cross correlation function reflect the Schwabe cycle with minima about every 11 years.

Summarising the data discussed so far we come to the following conclusions:

1. The ${ }^{10} \mathrm{Be}$ concentration reflects the sunspot cycle for the period $1750-1985$ quite well as far as the short-term (10-year scale) variations and the long-term (100-year scale) trend are concerned. However, there are three events (indicated in Figure 3) where an additional ${ }^{10} \mathrm{Be}$ peak appears.

2. The Fourier spectrum of the whole ${ }^{10} \mathrm{Be}$ record reveals a clear and sharp peak at 10.83 years with the second, third and fifth harmonic. This is in excellent agreement with the sunspot record which reveals a periodicity of 10.9 years for the same interval 1750 1985. The sharpness of the peak clearly indicates that in spite of the varying lengths 
of individual Schwabe cycles the mean cycle length seems to be precisely defined (see discussion of clock problem).

\subsection{Solar minima}

Periods of very low sunspot numbers are especially interesting. The most famous one is the Maunder Minimum period (1645 - 1715 A.D.) when almost no sunspots have been observed (Eddy 1976). The Spörer Minimum (1415-1535 A.D.) is only partly covered by our data. Its evidence is based mainly on ${ }^{14} \mathrm{C}$ measurements on tree rings (Stuiver \& Quay 1980).

During the Dalton Minimum (1795-1830 A.D.) sunspots were not completely absent but the numbers were relatively low.

${ }^{14} \mathrm{C}$ measurements on tree rings (Stuiver \& Quay 1980) and earlier ${ }^{10} \mathrm{Be}$ measurements on ice cores (Beer et al. 1991) revealed higher concentrations of these isotopes during the Maunder and Spörer minima which are in agreement with reduced solar activity. However, the strong damping in the carbon system of short-term variations and the limited time resolution of the ${ }^{10} \mathrm{Be}$ records made a detailed investigation of the Schwabe cycle during these periods impossible. The present ${ }^{10} \mathrm{Be}$ data clearly show that cyclic processes of the same frequency as the Schwabe cycle were operating during the minima (Figure 1(b)). There are indications that the amplitude of such processes were even larger than during epochs with high sunspot activity. This result is consistent with the observation that during the Maunder Minimum period auroras did not disappear.

\subsection{The "clock" problem}

Based on an analysis of the Schwabe cycle, Dicke (1978) raised the question whether the sunspot cycle is driven by a precise clock deeply buried inside the Sun meaning that a constant frequency is being phase modulated by a variable lag-function. Depending on the propagation time of each stroke to the solar surface the observable cycle length is changing. Nevertheless, according to this model the frequency averaged over many cycles is kept constant. The eruption model assumes that, if an eruption should be late, the lateness will be passed on to all the following cycles leading to a random walk in phase. One way to look at this question is to calculate the "melody", the momentary frequency for all times in a record. This has been done for the ${ }^{10} \mathrm{Be}$ record based on band-passed data (6-20 years). The results are shown in Figure 6.

The mean frequency of the melody is 0.0928 year $^{-1}$ corresponding to a periodicity of 10.78 years in excellent agreement with the power spectrum mean frequency of the Schwabe peak. Although the frequency shows fluctuations caused by changes in the phase, the mean phase shift is in agreement with the clock model. This result is consistent with the sharpness of the Schwabe cycle peak in the power spectrum (Figure 2). However, the record is still too short for a final answer. We cannot exclude that during earlier periods the phase shows a random walk. Other records also show remarkably sharp lines with the same frequencies supporting the hypothesis that the Schwabe cycle is a phase modulated oscillation (Mende, in preparation).

\subsection{Changes of solar irradiance and climate}

Satellite observations since 1978 show that the solar irradiance varies during a solar cycle due to fluctuations of the energy transport within the convective zone. However, the measured variations over one solar cycle are very small $(\sim 0.1 \%)$. Therefore, for a discussion of the relevance to the climate four basic questions have to be answered:

1) What is the potential variability of the solar irradiance? 2) How much did the irradi- 


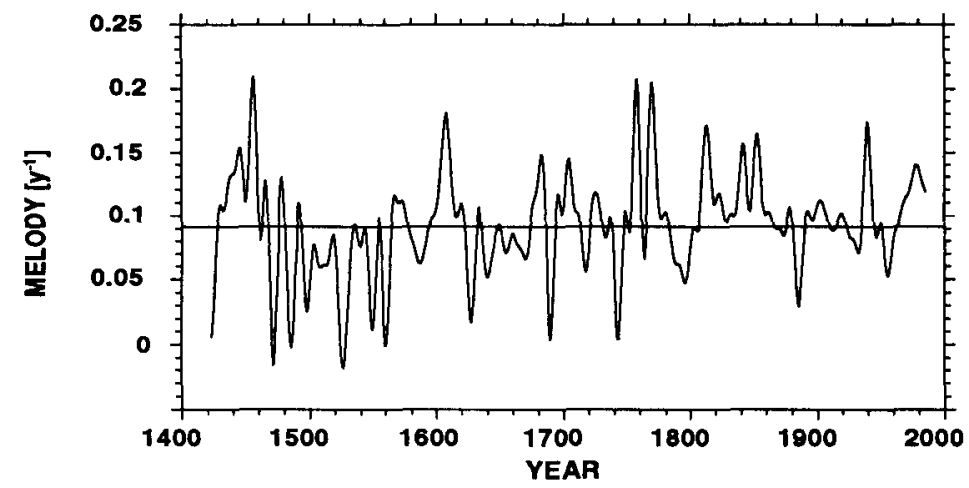

Figure $6 .{ }^{10} \mathrm{Be}$ melody derived from the ${ }^{10} \mathrm{Be}$ concentration after applying a band filter $(6-20 \mathrm{y})$. The mean frequency corresponds to a periodicity of $10.78 \mathrm{y}$ in excellent agreement with the 10.83 y peak in the Fourier spectrum (Figure 2).

ance change in the past? 3) How can we reconstruct these changes in the past? 4) What is the response of the climate system to such changes?

Based on a 25 year observation period of 33 Sun-like stars it has been concluded that the potential for brightness variability of the Sun is much larger than observed in the measurements since 1978 (Baliunas \& Jastrow 1990; Lockwood et al. 1992). Compared to the energy content of the convective zone of approximately $10^{45} \mathrm{ergs}$, a change in the solar irradiance by $0.5 \%$ for one century corresponds to a change of $10^{-4}$ of the total energy storage. The potential of solar variability seems to be much larger than executed during the last millennium.

A complete discussion of these topics is beyond the scope of this paper. We concentrate therefore mainly on question three. A large number of publications (Stephenson 1988) deals with the question how proxies of solar variability can be used to reconstruct changes in the irradiance. Since there are no long records of direct observations available, the only possible approach is to compare proxies with climate records, preferably the mean global surface temperature. However, this approach is hampered by several unknowns:

The quality of the older climate data is limited, because they are increasingly based on proxies. The response of the climate system to long-term trends has to be estimated with models. The measured relationship between solar variability and irradiance is only based on the very short observation period since 1978 and the underlying physical processes are not yet understood. The climate system is also strongly influenced by the increase in greenhouse gases and by changes of the atmospheric aerosol content.

In spite of all these difficulties many studies (Friis-Christensen \& Lassen 1991; Kelly \& Wigley 1992; Lean et al. 1992; Schlesinger \& Ramankutty 1992) have shown partially striking similarities between climate records and solar proxies.

In Figure 7 the inverted ${ }^{10} \mathrm{Be}$ record (Figure $1(b)$ ) is compared to a combined temperature record of the northern hemisphere (Groveman \& Landsberg 1979 and Jones et al. 1986). There is generally good agreement between the long-term trends. Also, some of the most prominent features such as the temperature minimum at 1815 and the subsequent sudden increase are well represented in the ${ }^{10} \mathrm{Be}$ record. During the 20th century the temperature increases more steeply than the ${ }^{10} \mathrm{Be}$ record. This could be interpreted as a result of greenhouse warming. On the basis of this simple correlation there is good reason to believe that the solar irradiance was changing in the past and that these changes are reflected in climate records. But for a quantitative discussion 


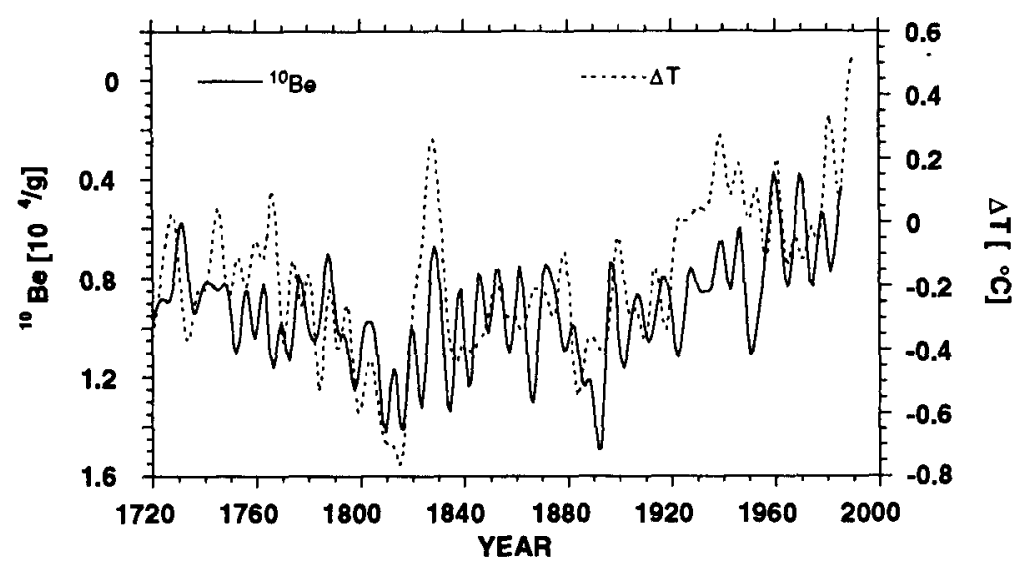

Figure 7. Comparison of the ${ }^{10} \mathrm{Be}$ record (Figure $1(b)$ ) with deviations from the mean temperature of the northern hemisphere (Groveman \& Landsberg 1979; Jones et al. 1986).

additional parameters like solar cycle lengths and atmospheric aerosol content have to be included.

\section{Summary and conclusions}

To date the Dye $3{ }^{10} \mathrm{Be}$ record provides the most impressive record of historical solar activity. It will be extended back by several thousand years in the near future. The analysis of the annual ${ }^{10} \mathrm{Be}$ concentration data covering the period 1423 to 1985 A.D. reveals interesting results concerning the solar-terrestrial relationship and provides the following answers to the questions raised by Newkirk (1984): The 11-year cycle does continue through the Maunder and most probably the Spörer Minimum. Although the cycle length varies deviations from the mean cycle length of 10.83 years seem to be compensated. The 87 -year Gleissberg cycle is also present in the ${ }^{10} \mathrm{Be}$ record.

There are clear indications that at least in part climate changes are related to changes in solar activity. An important question to be studied in more detail is the coupling between the solar activity and the Earth system. The relatively large variations of the UV part of the solar spectrum during a Schwabe cycle leads to large effects in the upper atmosphere which may be coupled in some, yet unknown way, to the lower atmosphere.

Acknowledgments. The authors thank all their colleagues involved in the core drilling, the sample preparation and the AMS measurements. J. Beer is especially indebted to Gordon Newkirk for his encouragement to work in this field. This work was supported by the Swiss National Science Foundation.

\section{REFERENCES}

Baliunas, S. \& Jastrow, R. 1990 Evidence for long-term brightness changes of solar-type stars. Nature 348, 520-523.

Beer, J., Blinov, A., Bonani, G., Finkel, R. C., Hofmann, H. J., Lehmann, B., Oeschger, H., Sigg, A., Schwander, J., Staffelbach, T., Stauffer, B., Suter, M. \& WoElfl, , W. 1990 Use of ${ }^{10} \mathrm{Be}$ in polar ice to trace the 11-year cycle of solar activity. Nature 347, 164-166. 
Beer, J., Raisbeck, G. M. \& Yiou, F. 1991 Time variations of ${ }^{10} \mathrm{Be}$ and solar activity. In The Sun in Time (ed. C. P. Sonett, M. S. Giampapa \& M. S. Matthews). pp. 343-359. Univ. of Arizona Press.

Buinov, A. 1988 The dependence of cosmogenic isotope production rate on solar activitiy and geomagnetic field variations. In Secular Solar and Geomagnetic Variations in the last 10,000 Years (ed. F. R. Stephenson \& A. W. Wolfendale). pp. 329-340. Kluwer Academic Publishers.

DICKE, R. H. 1978 Is there a chronometer hidden deep in the Sun? Nature 276, 676-680.

EDDY, J. A. 1976 The Maunder Minimum. Science 192, 1189-1201.

Frits-Christensen, E. \& Lassen, K. 1991 Length of the solar cycle: An indicator of solar activity closely associated with climate. Nature 254, 698-700.

Groveman, B. S. \& Landsberg H. E. 1979 Reconstruction of Northern hemisphere temperature. 1579 - 1880. University of Maryland, College Park, MD: Publication 79-181.

Jones, P. D., Raper, S. C. B., Bradley, R. S., Diaz, H. F., Kelly, P. M. \& Wigley, T. M. L. 1986 Northern Hemisphere surface air temperature variations, 1851-1984. J. Clim. Appl. Met. 25, 161-179.

Kelly, P. M. \& Wigley, T. M. L. 1992 Solar cycle length, greenhouse forcing and global climate. Nature 360, 328-330.

LAL, D. \& Peters, B. 1967 Cosmic ray produced radioactivity on the Earth. In Handbuch für Physik, 46/2 (ed. S. Flügge). pp. 551-612.

LEAN, J. 1991 Variations in the Sun's radiative output. Reviews of Geophysics 29/4, 505-535.

Lean, J., Hulburt, E. O., Skumanich, A. \& White, O. 1992 Estimating the Sun's radiative output during the Maunder Minimum. GRL 19, 1591-1594.

Lockwood, G.W., Skiff, B. A., Baliunas, S. L. \& Radick, R. R. 1992 Long-term solar brightness changes estimated from a survey of Sun-like stars. Nature 360, 653-655.

NewkIRK, G. JR. 1984 What accelerator mass spectrometry can do for solar physics. Nucl. Instrum. Meth. B5, 404-410.

RAISBECK, G. M. \& Yiou, F. $1980{ }^{10}$ Be in polar ice cores as a record of solar activity. In Proc. Conf. on Ancient Sun (ed. R.O. Pepin, J.A. Eddy \& R.B. Merrill). pp. 185-190. Pergamon Press.

Schlesinger, M. E. \& Ramankutty, N. 1992 Implications for global warming of intercycle solar irradiance variations. Nature 360, 330-333.

Siegenthaler, U. \& Beer, J. 1988 Model comparison of ${ }^{14} \mathrm{C}$ and ${ }^{10} \mathrm{Be}$ isotope records. In Secular Solar and Geomagnetic Variations in the last 10,000 Years (ed. F. R. Stephenson \& A. W. Wolfendale). pp. 315-328. Kluwer Academic Publishers.

Stephenson, F. R. 1988 Solar variability from historical records. In Secular Solar and Geomagnetic Variations in the last 10,000 Years (ed. F. R. Stephenson \& A. W. Wolfendale). pp. 109-129. Kluwer Academic Publishers.

Stuiver, M., Brazunias, T.F., Becker,B. \& Kromer, B. 1991 Climatic, solar, oceanic and geomagnetic influences on Late-Glacial and Holocene atmospheric $14 \mathrm{C} / 12 \mathrm{C}$ change. Quat. Res. 35, 1-24.

Stuiver, M. \& QuAY, P. D. 1980 Changes in atmospheric Carbon-14 attributed to a variable Sun. Science 207, 11-19.

Suter, M. 1990 Accelerator mass spectrometry: State of the art in 1990. Nucl. Instrum. Meth. B52, 211-223.

Willson, R.C. \& Hudson, H. S. 1988 Solar luminosity variations in solar cycle 21. Nature 332, 810-812. 